functions and conditions of equilibrium, while the second (214 pages) is concerned with the application to gases, gaseous equilibria, phase equilibria and solutions. The approach throughout is properly based on the chemical potential, and the treatment, always adequate, is at times illuminating. The student who works through these chapters will be amply rewarded; but work he will have to, for although care has been taken to ensure clarity, the style is at times ponderous and he may well get the impression that the subject is more difficult than it really is. He will be helped, however, by the illustrations in the text and by the graded problems at the end of each chapter - a very valuable feature of the book. Only by submitting himself to the rigid discipline of working examples does the student begin to appreciate the value and versatility of the thermodynamic tool and to acquire the confidence to use it in attacking practical problems. The chemistry student will find the treatment of cells and electrolytes too brief and will regret the exclusion of surface phenomena. The third part (150 pages), devoted to statistical mechanics, contains interesting chapters on the statistical analogues of entropy and free energy, the partition functions of gases, and crystals and the third law, but the beginner may find them above his head. The two final chapters, one giving a brief treatment of regular solutions and adsorption, and the other on some aspects of chemical kinetics, might well have been replaced by a statistical treatment of chemical equilibrium, which receives only scant attention. There is even no mention of the functions $\left(G^{0}-H^{0}{ }_{0}\right) / 0 \mathrm{~T}$ and $\left(\mathrm{H}^{0}-\mathrm{H}^{0}{ }_{0}\right)$ and of their use.

Prof. Denbigh's book will be useful to the brighter students and to teachers of the subject; but those applied chemists and chemical engineers who wish to be led gently up the thermodynamic slope may seek a less strenuous guide.

\section{TOWARDS THE NEW CYTOLOGY}

\section{Physiologie der Zelle}

Von Dr. Johannes Haas. Pp. 474. (Berlin-Nikolassee: Gebrüder Borntraeger, 1955.) 48 D.M.

$\mathrm{T}$ HE need for a text-book presenting an integrated account of cell organization in the light of developments of the past twenty-five years has for some time been evident, and Ir. J. Haas's book goes a long way towards satisfying this need. The modernity of its approach is indicated by an initial section on molecular morphology, accounting for about one-third of the volume. There is to this extent a certain parallel with Frey.Wyssling's book, "Submicroscopic Morphology of Protoplasm and its Derivatives"; but there is a wealth of difference between the two, for the emphasis in Dr. Haas's book is primarily biochemical. Within the compass of a hundred and sixty pages he examines the structure and properties of proteins, nucleic acids, enzymes and lipoids, and then proceeds to a consideration of cell organelles : cytoplasm, cell surface, mitochondria, nucleus and interphase-chromosomes (119 pp.). In a third section he discusses selected cell functions: respiration, exchanges with the environment, cell division, growth and differentiation (155 pp.).
The extent to which the author has succeeded in integrating a work of this scope is highly commend. able; but he has not managed to avoid repetition. It would be easy to draw up a list of references missed, and for British and American readers there will be some shocks; but these omissions do not impair the over-all achievement. They are more than offset by the number of references which will be new to many readers.

On a different plane of criticism, one may perhaps regret that Dr. Haas seems not entirely conscious of the revolution in ideas which has occurred and of which he displays the results. He states (p. 17) that the object of physiology is the study of function in its dependence on organic structure prevailing at a given time. It is to be doubted whether any physiologist of thirty years ago would have so expressed his aim; and there are probably many physiologists to-day who will be surprised by such a definition. For cell physiology, the definition is a concise statement of present aims; but Dr. Haas allows it to pass as if it were a commonplace. Related to this lack of historical awareness is a more serious criticism - the more serious since this book is clearly a textbook-namely, that he frequently introduces a new topic by a statement derived from classical cytological views, but made as if those views still retained their original force. Thus he quotes without comment (p. 170) Wilson's view that the structureless hyalo. plasm is the most constant and active component of the cell, even though he is well aware of the extent to which this opinion requires modification in the light of the role of the mitochondria. Again, he repeats-and does so as if the generalization admitted of no exceptions-that "the living nucleus appears optically empty, both in normal light and dark. ground". If students are to be spared the necessity of leaping old hurdles, it is the limited value of the light-microscopic image which requires emphasis; and what is important is that the nucleus is not optically homogeneous when viewed by phasecontrast. The reason for such statements is the difficulty we all experience of escaping from the somewhat mystical concepts of classical cytology : the twin man-made ghosts, the nucleus and the cytoplasm, both homogeneous in the light-microscope-and by implication homogeneous in every other respect as well.

Not only in cytological matters, but also in the exposition of biochemical topics, Dr. Haas sometimes modifies his initial position profoundly and disconcertingly at a later stage. Thus, the discussion of molecular shapes (p. 28 et seq.) proceeds as if chain molecules of the linear polypeptide or polysaccharide type were the only type of linear macromolecules known. Not until the discussion of cytoplasmic structure (p. 194) do we learn of polymerization of globular molecules to form fibrils.

Dr. Haas writes a simple German style of somewhat unusually short sentences. Particularly successful sections are those on mitochondria, nucleus, cellrespiration, growth and differentiation. It is evident that considerations of cost have influenced both layout and the extreme economy of illustration. The latter is regrettable ; for Dr. Haas's book, in spite of its title of 'cell physiology', is a 'cytology'. That is one indication of the distance travelled during the past twenty-five years; another is this, that the name of Boveri occurs but once, and without a reference.

Laurenct Prcken 